\title{
A list of nearby dwarf galaxies towards the Local Void in Hercules-Aquila
}

\author{
V.E. Karachentseva ${ }^{1,2}$, I.D. Karachentsev ${ }^{1,3}$, and G.M. Richter ${ }^{4}$ \\ 1 Visiting astronomer, Astrophysikalisches Institut Potsdam, An der Sternwarte 16, D-14482 Potsdam, Germany \\ 2 Astronomical Observatory of Kiev University, Observatorna 3, 254053, Kiev, Ukraine \\ 3 Special Astrophysical Observatory, Russian Academy of Sciences, N. Arkhyz, KChR, 357147, Russia \\ 4 Astrophysicalisches Institut Potsdam, an der Sternwarte 16, D-14482 Potsdam, Germany
}

Received July 6; accepted September 21, 1998

\begin{abstract}
Based on film copies of the POSS-II we inspected a wide area of $\sim 6000 \square^{\circ}$ in the direction of the nearest cosmic void: $\left\{\mathrm{RA}=18^{\mathrm{h}} 38^{\mathrm{m}}, D=+18^{\circ}, V_{0}<\right.$ $\left.1500 \mathrm{~km} \mathrm{~s}^{-1}\right\}$. As a result we present a list of 78 nearby dwarf galaxy candidates which have angular diameters $\gtrsim 0.5$ and a mean surface brightness $\lesssim 26 \mathrm{mag} / \square^{\prime \prime}$. Of them 22 are in the direction of the Local Void region. To measure their redshifts, a HI survey of these objects is undertaken on the $100 \mathrm{~m}$ Effelsberg telescope.
\end{abstract}

Key words: galaxies: general — galaxies: dwarf

\section{Introduction}

As it is well known now, empty cosmic volumes (=voids) are the most prominent features of the large scale structure of the Universe. Besides huge voids of about $100 \mathrm{Mpc}$ size which are revealed in deep galaxy redshift surveys (Joeveer et al. 1978; Kirshner et al. 1981; de Lapparent et al. 1986), in the very nearby Universe one can find also "minivoids" with a diameter of $3-5 \mathrm{Mpc}$ (Karachentsev 1994). Are the voids completely empty of galaxies? The question may be answered more easily by studying nearby voids.

Compiling the Catalogue and the Atlas of nearby galaxies which covers the Local Supercluster volume, Tully (1988) noted a wide sky region towards $\mathrm{RA} \sim 19^{\mathrm{h}}$, and $D \sim+20^{\circ}$ which is absolutely avoided by galaxies from known catalogues. Extinction at low galactic latitudes cannot be the main cause of this phenomenon. Unfortunately, Tully did not indicate even approximately the Local Void boundary. This led to a confusion in which different authors ascribed the same name to different volumes. Below we use our own definition of the center and extent of the Local Void.
Our main goal was to search for new nearby dwarf galaxies in this Local Void. Because the Local Void begins actually just beyond the Local Group edge, we obtain here unprecedently a low threshold for detection of very faint dwarf galaxies in a void, which is an order of magnitude lower than for detection in other voids.

\section{Field of the search}

To outline the position of the Local Void on the sky, we reproduce in Fig. 1 the distribution of galaxies with known corrected radial velocities $V_{0}<1500 \mathrm{~km} \mathrm{~s}^{-1}$ in a region $\mathrm{RA}=[10.0,23.5]^{\mathrm{h}}, D=[-20,+60]^{\circ}$. The 19 nearest galaxies with $V_{0}<750 \mathrm{~km} \mathrm{~s}^{-1}$ are shown as larger open circles. The radial velocities were taken from the PGC catalogue (Paturel et al. 1992) and updated with data from recent publications. The curves correspond to the Milky Way avoidance zone within galactic latitudes +10 and $-10^{\circ}$. As it can be seen from Fig. 1, inside a circle of $60^{\circ}$ diameter around a point of $\left\{\mathrm{RA}=18^{\mathrm{h}} 38^{\mathrm{m}}, D=\right.$ $\left.+18^{\circ}\right\}$ there is no galaxy having a radial velocity $V_{0}<$ $1500 \mathrm{~km} \mathrm{~s}^{-1}$. Searches for new nearby galaxies in the most obscured part of this area undertaken by Nakanishi et al. (1997) among the IRAS sources, as well for HI-rich dwarf galaxies (Hoffman et al. 1992), added nothing within the drawn circle.

The distribution of more distant galaxies in the same sky region is shown in Fig. 2. The first galaxies inside the Local Void zone appear just beyond $V_{0}=1500 \mathrm{~km} \mathrm{~s}^{-1}$. Adopting for the Hubble parameter $H=75 \mathrm{~km} \mathrm{~s}^{-1} / \mathrm{Mpc}$, we find that the empty volume is $>310^{3} \mathrm{Mpc}^{3}$.

It should be emphasized that there is still no systematic search for nearby dwarf galaxies in the whole Local Void area. Therefore, the possibility for such a large volume to be completely devoided of galaxies remains unproved. In the next section we describe the results of a 


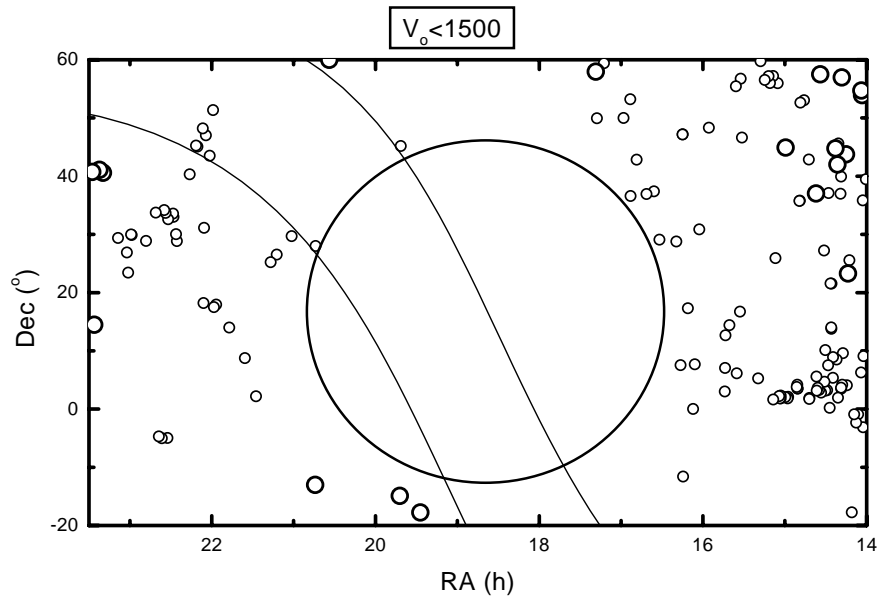

Fig. 1. Distribution of galaxies from PGC with radial velocities $V_{0}<1500 \mathrm{~km} \mathrm{~s}^{-1}$ in the equatorial coordinates. The nearest galaxies with corrected radial velocities $V_{0}<750 \mathrm{~km} \mathrm{~s}^{-1}$ are marked by larger circles. Two curves outline the Milky Way region with $|b|<10^{\circ}$. A circle of 60 degree diameter indicates the Hercules-Aquila void region

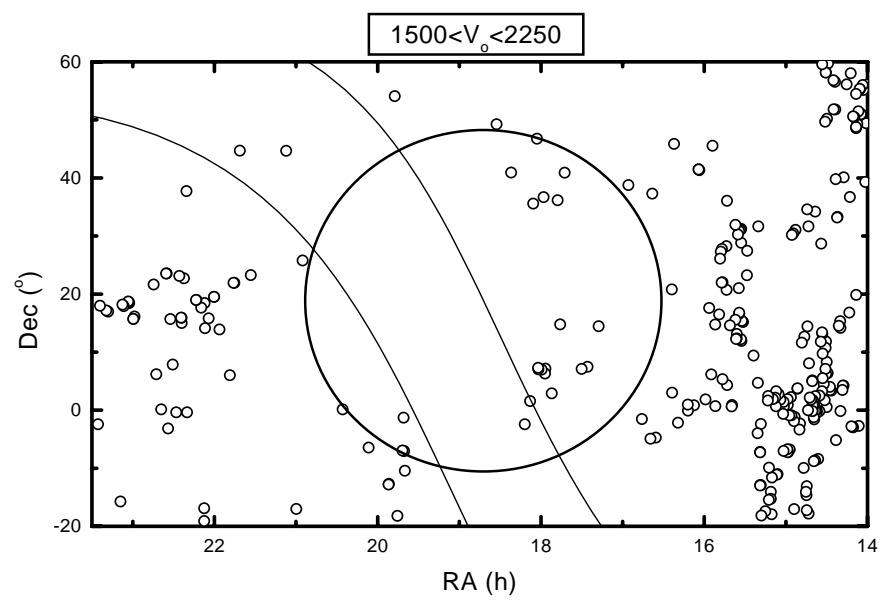

Fig. 2. The same as Fig. 1 but for more distant galaxies with $1500<V_{0}<2250 \mathrm{~km} \mathrm{~s}^{-1}$

special survey for nearby dwarf galaxy candidates on almost the whole area of the Local Void.

\section{The list of dwarf galaxy candidates}

To detect new dwarf galaxies we used $J$ and $R$ film copies of the Second Palomar Sky Survey, POSS-II. The high angular resolution of new photographic emulsions and their sensitivity to low contrast details permit the detection and classification with confidence of galaxies of small angular dimensions, especially of low surface brightness objects. Following the method described earlier (Karachentseva \& Karachentsev 1998 = Survey I), we searched for dwarf galaxies with angular diameters more than about 0.5 arcmin in a wide region limited to $\mathrm{RA}=[14.0,23.5]^{\mathrm{h}}$, $D=[-3,+63]^{\circ}$. Because in the previous Survey-I

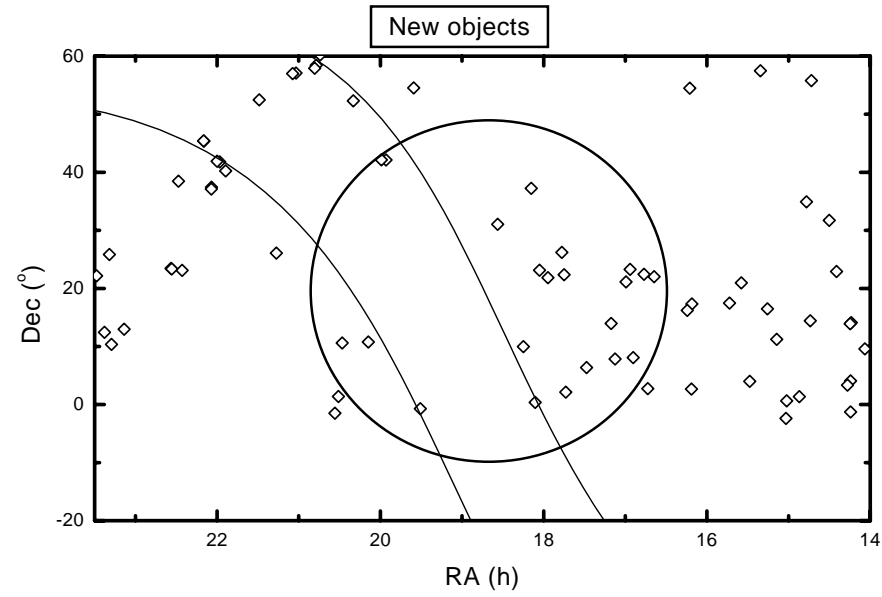

Fig. 3. Distribution of new dwarf galaxy candidates in the same area (diamonds)

we searched for very nearby dwarf galaxies with $V_{0}<$ $500 \mathrm{~km} \mathrm{~s}^{-1}$, i.e. about 3 times lower than in the present survey, we relaxed slightly our criterion, not requiring signs of possible resolution of into stars.

We found 78 dwarf galaxy candidates, which are presented in Table 1. Here Col. 1 gives the object number indicating the POSS-II field number; Col. 2 contain the equatorial coordinates (epoch 1950.0); Col. 3 gives the major and minor angular diameters in arcmin measured on the blue and red films, respectively; Col. 4 gives the morphological type in the usual designations ("d" as a "dwarf" was omitted); Col. 5 gives a rough estimate of surface brightness: H-high (equal or brighter than for a normal spiral galaxy), L — low, VL — very low, EL — extremely low, i.e. invisible on POSS-I (this means a mean SB brighter than about $\left.26 \mathrm{mag} / \square^{\prime \prime}\right)$; Col. 6 gives the crossidentification with other lists (D or F - Schombert et al. 1992, BCh, 83 - Balkowski \& Chamaraux 1983); Col. 7 gives the galaxy morphology description, or the heliocentric radial velocity and magnitude estimated from NED.

The distribution of the 78 galaxies from Table 1 is presented in Fig. 3 by diamonds. Obviously, the detected new objects are distributed more or less regularly over the whole studied area. This picture gives no indication of the Local Void. However, only redshift measurements for the new objects can show, if they fill the Local Void volume or not. A HI survey of the objects from Table 1 is presently in progress at the $100-\mathrm{m}$ Effelsberg radio telescope. (Huchtmeier 1998).

\section{Discussion}

As can be seen from Table 1, among 78 galaxy candidates, 59 are not in previous lists. For the 8 galaxies with known radial velocities the median is $+1690 \mathrm{~km} \mathrm{~s}^{-1}$, which shows that they are actually dwarfs. 
Table 1. List of new dwarf galaxy candidates towards the Local Void

\begin{tabular}{|c|c|c|c|c|c|c|c|c|}
\hline \multirow{3}{*}{$\begin{array}{l}\text { Object } \\
(1) \\
721.2\end{array}$} & $\overline{\mathrm{RA}}$ & 0) & \multicolumn{2}{|c|}{$\begin{array}{l}(a \times b)_{\mathrm{b}} \\
(a \times b)_{\mathrm{r}}\end{array}$} & Type & SB & Identification & Notes \\
\hline & \multicolumn{2}{|c|}{$(2)$} & \multicolumn{2}{|c|}{$(3)$} & $(4)$ & $(5)$ & $(6)$ & $\overline{(7)}$ \\
\hline & \multirow[t]{2}{*}{$13^{\mathrm{h}} 55^{\mathrm{m}} 16^{\mathrm{s}} 8$} & \multirow[t]{2}{*}{$+08^{\circ} 04^{\prime} 51^{\prime \prime}$} & 0.50 & 0.30 & Ir & $\mathrm{L}$ & \multirow[t]{2}{*}{ D721-17 } & \\
\hline & & & 0.60 & 0.45 & & & & \\
\hline \multirow{2}{*}{721.3} & \multirow[t]{2}{*}{140358.2} & \multirow{2}{*}{ +093543 } & 0.85 & 0.25 & $\operatorname{Im}$ & $\mathrm{L}$ & \multirow[t]{4}{*}{ D721-03 } & comet-like \\
\hline & & & 0.80 & 0.30 & & & & \\
\hline \multirow[t]{2}{*}{384.4} & \multirow[t]{2}{*}{140501.5} & \multirow[t]{2}{*}{+351747} & 1.10 & 0.65 & Ir & $\mathrm{L}$ & & resolved?, blue \\
\hline & & & 1.00 & 0.60 & & & & \\
\hline \multirow[t]{2}{*}{650.2} & 141357.0 & +140624 & 1.10 & 1.10 & PN? & $\mathrm{VL}$ & F650-01 & \\
\hline & & & 0.80 & 0.80 & & & & \\
\hline 794.1 & 141426.1 & +040355 & 1.00 & 0.65 & Ir & $\mathrm{L}$ & $\mathrm{BCh}, 83$ & patchy \\
\hline & & & 0.90 & 0.45 & & & & \\
\hline 866.1 & 141428.9 & -011624 & 1.00 & 0.50 & Ir & $\mathrm{L}$ & & patchy \\
\hline & & & - & - & & & & \\
\hline 650.1 & 141444.3 & +135459 & 0.60 & 0.50 & Ir & $\mathrm{L}$ & & \\
\hline & & & 0.55 & 0.50 & & & & \\
\hline 794.2 & 141645.4 & +032108 & 1.30 & 0.80 & $\mathrm{Ir} ?$ & $\mathrm{~L}$ & BCh, 83 & \\
\hline & & & - & - & & & & \\
\hline 511.1 & 142447.9 & +225451 & 0.90 & 0.60 & Sph? & $\mathrm{VL}$ & & \\
\hline & & & 0.80 & 0.60 & & & & \\
\hline 447.2 & 143010.3 & +314345 & 0.65 & 0.50 & $\mathrm{Ir}$ & $\mathrm{L}$ & & \\
\hline & & & 0.65 & 0.50 & & & & \\
\hline 176.3 & 144314.7 & +554756 & 0.80 & 0.55 & Ir & $\mathrm{L}$ & & \\
\hline & & & - & - & & & & \\
\hline 651.1 & 144401.3 & +142518 & 1.20 & 0.75 & Ir & $\mathrm{L}$ & F651-2 & $V_{\mathrm{h}}=1789$ \\
\hline & & & 1.15 & 0.65 & & & & \\
\hline 386.1 & 144650.0 & +345458 & 1.10 & 0.90 & $\mathrm{Sm} ?$ & $\mathrm{~L}$ & UGC 9540 & \\
\hline & & & - & - & & & & \\
\hline 868.3 & 145209.8 & +012150 & 0.50 & 0.30 & $\mathrm{Ir}$ & $\mathrm{L}$ & (ISI, 96) & distant?, $17.8^{\mathrm{m}}$ \\
\hline & & & - & - & & & & \\
\hline 868.2 & 150122.7 & +003725 & 0.50 & 0.35 & $\mathrm{Ir}$ & $\mathrm{L}$ & & red knot \\
\hline & & & - & - & & & & \\
\hline 868.1 & 150158.2 & -022335 & 1.00 & 0.70 & Ir & $\mathrm{H}$ & & \\
\hline & & & - & - & & & & \\
\hline 724.1 & 150846.1 & +11 1313 & 0.45 & 0.30 & Ir & $\mathrm{L}$ & & bluish \\
\hline & & & 0.40 & 0.30 & & & & \\
\hline 653.1 & 151535.5 & +162927 & 0.50 & 0.45 & $\mathrm{Ir}$ & $\mathrm{H}$ & MCG3-39-9 & blue, $15.7^{\mathrm{m}}$ \\
\hline & & & 0.50 & 0.45 & & & & \\
\hline 171.3 & 152044.7 & +572917 & 0.90 & 0.60 & Ir & VL & & blue \\
\hline & & & - & - & & & & \\
\hline 797.2 & 152836.0 & +035958 & 1.30 & 0.90 & $\mathrm{Sm}$ & $\mathrm{L}$ & & distant? \\
\hline & & & 1.20 & 1.00 & & & & \\
\hline 582.1 & 153447.4 & +205731 & 1.1: & $0.8:$ & $\mathrm{SB} ?$ & $\mathrm{~L}$ & F583-2 & $V_{\mathrm{h}}=1720$ \\
\hline & & & 1.1: & $0.8:$ & & & & \\
\hline 583.1 & 154327.7 & +172804 & 1.50 & 1.00 & $\mathrm{SB} ?$ & $\mathrm{~L}$ & F583-5 & $15.83^{\mathrm{m}}$ \\
\hline & & & - & - & & & & \\
\hline 655.1 & 161105.1 & +171907 & 1.40 & 0.90 & $\mathrm{Sm}$ & $\mathrm{L}$ & UGC 10281 & $V_{\mathrm{h}}=1080$ \\
\hline & & & 1.30 & 0.80 & & & & \\
\hline 799.1 & 161125.5 & +023920 & 0.70 & 0.50 & Ir & $\mathrm{L}$ & & \\
\hline & & & 0.70 & 0.50 & & & & \\
\hline 178.2 & 161237.3 & +542946 & 1.10 & 0.65 & Ir & $\mathrm{L}$ & & blue \\
\hline & & & - & - & & & & \\
\hline 656.1 & 161428.2 & +16 1230 & 0.45 & 0.40 & Ir & $\mathrm{L}$ & & \\
\hline & & & 0.40 & 0.35 & & & & \\
\hline 586.1 & 163847.8 & +220045 & 0.40 & 0.20 & $\mathrm{Ir} ?$ & $\mathrm{~L}$ & & near a bright \\
\hline & & & 0.35 & 0.20 & & & & star \\
\hline
\end{tabular}


Table 1. continued

\begin{tabular}{|c|c|c|c|c|c|c|c|c|}
\hline (1) & & & & & $(4)$ & $(5)$ & $(6)$ & $(7)$ \\
\hline 801.1 & 164330.2 & +024439 & $\begin{array}{l}1.00 \\
-\end{array}$ & $\begin{array}{l}0.30 \\
-\end{array}$ & Ir? & VL & & $\begin{array}{l}\text { bright nucl. }+ \\
\text { blue halo }\end{array}$ \\
\hline 586.2 & 164613.8 & +222527 & $\begin{array}{l}0.70 \\
0.55\end{array}$ & $\begin{array}{l}0.35 \\
0.35\end{array}$ & SB? & $\mathrm{L}$ & & blue \\
\hline 802.3 & 165413.8 & +080434 & $\begin{array}{l}1.10 \\
0.85\end{array}$ & $\begin{array}{l}0.85 \\
0.50\end{array}$ & $\mathrm{Sm} ?$ & VL & & \\
\hline 518.1 & 165627.4 & +231650 & $\begin{array}{l}0.70 \\
0.55\end{array}$ & $\begin{array}{l}0.35 \\
0.30\end{array}$ & Ir & $\mathrm{L}$ & & smoothed, blue \\
\hline 587.1 & 165925.4 & +210755 & $\begin{array}{l}0.45 \\
-\end{array}$ & $\begin{array}{l}0.40 \\
-\end{array}$ & Ir & $\mathrm{L}$ & & \\
\hline 802.2 & 170731.2 & +075055 & $\begin{array}{l}2.10 \\
1.70\end{array}$ & $\begin{array}{l}0.90 \\
0.70\end{array}$ & $\mathrm{Sm}$ & $\mathrm{L}$ & & no nuclea \\
\hline 659.1 & 171024.5 & +135758 & $\begin{array}{l}0.50 \\
0.40\end{array}$ & $\begin{array}{l}0.30 \\
0.30\end{array}$ & Ir & $\mathrm{L}$ & & blue \\
\hline 804.6 & 172826.6 & +062225 & $\begin{array}{l}0.45 \\
-\end{array}$ & $\begin{array}{l}0.30 \\
-\end{array}$ & Ir & $\mathrm{L}$ & & $\begin{array}{l}\text { comp.? of N6384 } \\
\text { having } V_{\mathrm{h}}=1665\end{array}$ \\
\hline 804.1 & 174345.0 & +020804 & $\begin{array}{l}1.10 \\
-\end{array}$ & $\begin{array}{l}0.75 \\
-\end{array}$ & $\mathrm{Sm} ?$ & $\mathrm{~L}$ & & \\
\hline 589.3 & 174457.1 & +222147 & $\begin{array}{l}0.70 \\
0.60\end{array}$ & $\begin{array}{l}0.40 \\
0.35\end{array}$ & Ir & $\mathrm{L}$ & & \\
\hline 521.2 & 174640.7 & +261301 & $\begin{array}{l}0.80 \\
0.75\end{array}$ & $\begin{array}{l}0.45 \\
0.40\end{array}$ & Ir & $\mathrm{L}$ & F520-3 & \\
\hline 589.2 & 175653.7 & +215102 & $\begin{array}{l}0.60 \\
0.40\end{array}$ & $\begin{array}{l}0.35 \\
0.25\end{array}$ & Ir & VL & & \\
\hline 521.1 & 180301.7 & +230812 & $\begin{array}{l}1.30 \\
1.10\end{array}$ & $\begin{array}{l}0.90 \\
0.70\end{array}$ & Ir & $\mathrm{L}$ & & $\begin{array}{l}\text { near } \mathrm{U} 11111 / 13 \\
\text { with } V_{\mathrm{h}}=2550\end{array}$ \\
\hline 877.2 & 180614.5 & +002210 & $\begin{array}{l}- \\
1.80\end{array}$ & $\begin{array}{l}- \\
1.60\end{array}$ & PN? & $\mathrm{EL}$ & & near PN18034+0022 \\
\hline 335.1 & 180909.0 & +371409 & $\begin{array}{l}0.45 \\
0.40\end{array}$ & $\begin{array}{l}0.40 \\
0.35\end{array}$ & $\mathrm{Ir}$ & $\mathrm{L}$ & & distant? \\
\hline 734.1 & 181454.8 & +095804 & $\begin{array}{l}0.70 \\
0.60\end{array}$ & $\begin{array}{l}0.60 \\
0.50\end{array}$ & $\mathrm{Sm} ?$ & $\mathrm{~L}$ & & distant? \\
\hline 457.1 & 183348.0 & +310218 & $\begin{array}{l}0.70 \\
-\end{array}$ & $\begin{array}{l}0.60 \\
-\end{array}$ & $\mathrm{Ir}$ & $\mathrm{L}$ & & \\
\hline 882.1 & 193040.0 & -004345 & $\begin{array}{l}1.00 \\
0.90\end{array}$ & $\begin{array}{l}0.90 \\
0.80\end{array}$ & $\mathrm{Ir}$ & $\mathrm{L}$ & CGMW 3-4255 & Roman et al., 96 \\
\hline 185.1 & 193528.2 & +543133 & $\begin{array}{l}0.85 \\
-\end{array}$ & $\begin{array}{l}0.65 \\
-\end{array}$ & $\mathrm{Ir}$ & $\mathrm{L}$ & & \\
\hline 284.2 & 195554.0 & +420734 & $\begin{array}{l}0.85 \\
0.65\end{array}$ & $\begin{array}{l}0.45 \\
0.40\end{array}$ & Ir & $\mathrm{L}$ & & \\
\hline 142.3 & 195714.7 & +622909 & $\begin{array}{l}0.90 \\
0.70\end{array}$ & $\begin{array}{l}0.45 \\
0.45\end{array}$ & Ir & $\mathrm{L}$ & & patchy, bluish \\
\hline 284.1 & 195913.1 & +420905 & $\begin{array}{l}0.70 \\
0.65\end{array}$ & $\begin{array}{l}0.35 \\
0.35\end{array}$ & $\mathrm{Ir}$ & $\mathrm{L}$ & & \\
\hline 740.1 & 200849.0 & +104642 & $\begin{array}{l}0.60 \\
0.60\end{array}$ & $\begin{array}{l}0.40 \\
0.40\end{array}$ & Ir & $\mathrm{L}$ & & bluish \\
\hline 233.1 & 201952.6 & +521826 & $\begin{array}{l}0.75 \\
0.50\end{array}$ & $\begin{array}{l}0.35 \\
0.20\end{array}$ & Ir & $\mathrm{L}$ & & distant? \\
\hline 741.1 & 202805.4 & +103556 & $\begin{array}{l}0.80 \\
0.80\end{array}$ & $\begin{array}{l}0.20 \\
0.20\end{array}$ & Ir & VL & & smoothed \\
\hline 884.3 & 203049.1 & +012335 & $\begin{array}{l}0.65 \\
0.55\end{array}$ & $\begin{array}{l}0.30 \\
0.30\end{array}$ & $\operatorname{Ir} ?$ & $\mathrm{~L}$ & & distant? \\
\hline 885.2 & 203323.7 & -012925 & $\begin{array}{l}0.75 \\
0.75\end{array}$ & $\begin{array}{l}0.30 \\
0.25\end{array}$ & Ir & $\mathrm{L}$ & & distant? \\
\hline 144.2 & 204415.2 & +601340 & $\begin{array}{l}0.60 \\
-\end{array}$ & $\begin{array}{l}0.40 \\
-\end{array}$ & Ir & $\mathrm{L}$ & & $\begin{array}{l}\text { comet-like, } \\
\text { comp. N6946? }\end{array}$ \\
\hline 144.1 & 204711.5 & +582556 & $\begin{array}{l}0.70 \\
- \\
\end{array}$ & $\begin{array}{l}0.45 \\
- \\
\end{array}$ & $\mathrm{Ir}$ & $\mathrm{L}$ & & comp. N6946? \\
\hline
\end{tabular}


Table 1. continued

\begin{tabular}{|c|c|c|c|c|c|c|c|c|}
\hline (1) & \multicolumn{2}{|c|}{$(2)$} & \multicolumn{2}{|c|}{$(3)$} & $(4)$ & $(5)$ & $(6)$ & (7) \\
\hline 144.5 & 204636.5 & +625303 & $\begin{array}{l}0.50 \\
-\end{array}$ & $\begin{array}{l}0.45 \\
-\end{array}$ & $\mathrm{Ir}$ & $\mathrm{L}$ & & $\begin{array}{l}3 \text { knots at SW, } \\
\text { comp. N6946?? }\end{array}$ \\
\hline 144.3 & 204819.1 & +575504 & $\begin{array}{l}2.10 \\
-\end{array}$ & $\begin{array}{l}0.20 \\
-\end{array}$ & $\mathrm{Sm}$ & $\mathrm{VL}$ & IRAS & $\begin{array}{l}\text { edge-on, } \\
V_{\mathrm{h}}=2763\end{array}$ \\
\hline 144.4 & 210202.8 & +570517 & $\begin{array}{l}2.30 \\
-\end{array}$ & $\begin{array}{l}1.40 \\
-\end{array}$ & Ir & $\mathrm{VL}$ & & $\begin{array}{l}\text { isolated cirrus? } \\
\text { knots? }\end{array}$ \\
\hline 144.6 & 210430.6 & +570015 & $\begin{array}{l}0.70 \\
-\end{array}$ & $\begin{array}{l}0.50 \\
-\end{array}$ & Ir & VL & & \\
\hline 530.1 & 211629.6 & +260456 & $\begin{array}{l}1.00 \\
0.80\end{array}$ & $\begin{array}{l}0.65 \\
0.60\end{array}$ & $\mathrm{Sm} ?$ & $\mathrm{~L}$ & & distant? \\
\hline 236.1 & 212904.3 & +522824 & $\begin{array}{l}- \\
1.10\end{array}$ & $\begin{array}{l}- \\
0.60\end{array}$ & Ir? & EL & Dw095+1.0? & $\begin{array}{l}V_{\mathrm{h}}=+159, \text { Henning } \\
\text { et al., } 98\end{array}$ \\
\hline 344.1 & 215340.8 & +401405 & $\begin{array}{l}0.60 \\
0.60\end{array}$ & $\begin{array}{l}0.35 \\
0.35\end{array}$ & Ir & $\mathrm{L}$ & & smoothed \\
\hline 344.3 & 215805.5 & +414715 & $\begin{array}{l}1.0: \\
0.6\end{array}$ & $\begin{array}{l}0.6 \\
0.45\end{array}$ & $\mathrm{Ir}$ & $\mathrm{VL}$ & & structured \\
\hline 344.2 & 220002.0 & +415427 & $\begin{array}{l}0.60 \\
0.50\end{array}$ & $\begin{array}{l}0.40 \\
0.40\end{array}$ & Ir & $\mathrm{L}$ & & \\
\hline 404.2 & 220408.9 & +372525 & $\begin{array}{l}1.00 \\
-\end{array}$ & $\begin{array}{l}0.60 \\
-\end{array}$ & Ir? & VL & & \\
\hline 404.1 & 220412.3 & +370631 & $\begin{array}{l}0.45 \\
-\end{array}$ & $\begin{array}{l}0.30 \\
-\end{array}$ & Ir? & $\mathrm{L}$ & & blue \\
\hline 289.2 & 220943.2 & +452145 & $\begin{array}{l}2.00 \\
1.80\end{array}$ & $\begin{array}{l}1.40 \\
1.20\end{array}$ & $\mathrm{Sm}$ & $\mathrm{L}$ & & \\
\hline 289.3 & 220954.9 & +452512 & $\begin{array}{l}0.70 \\
0.55\end{array}$ & $\begin{array}{l}0.40 \\
0.35\end{array}$ & Ir & $\mathrm{L}$ & & \\
\hline 602.4 & 222544.2 & +230708 & $\begin{array}{l}0.65 \\
0.60\end{array}$ & $\begin{array}{l}0.55 \\
0.55\end{array}$ & $\mathrm{Ir}$ & $\mathrm{L}$ & F533-1 & $V_{\mathrm{h}}=1278$ \\
\hline 345.1 & 222824.7 & +382830 & $\begin{array}{l}1.2: \\
0.90\end{array}$ & $\begin{array}{l}0.80 \\
0.60\end{array}$ & Ir & $\mathrm{VL}$ & & \\
\hline 534.1 & 223348.4 & +232702 & $\begin{array}{l}0.75 \\
0.70\end{array}$ & $\begin{array}{l}0.55 \\
0.55\end{array}$ & Ir? & $\mathrm{L}$ & F533-v1 & $17.24^{\mathrm{m}}$ \\
\hline 534.2 & 223317.5 & +232050 & $\begin{array}{l}0.35 \\
0.30\end{array}$ & $\begin{array}{l}0.35 \\
0.30\end{array}$ & Sph? & $\mathrm{VL}$ & & $\begin{array}{l}\text { comp.(?) of a pair } \\
\text { N7332/9 w. } V_{\mathrm{h}}=1300\end{array}$ \\
\hline 748.3 & 230819.5 & +125801 & $\begin{array}{l}1.10 \\
1.00\end{array}$ & $\begin{array}{l}0.65 \\
0.50\end{array}$ & Ir & VL & & \\
\hline 749.1 & 231739.6 & +102057 & $\begin{array}{l}0.60 \\
0.55\end{array}$ & $\begin{array}{l}0.35 \\
0.35\end{array}$ & Ir & $\mathrm{L}$ & & knots \\
\hline 536.1 & 231909.1 & +254955 & $\begin{array}{l}0.65 \\
0.25\end{array}$ & $\begin{array}{l}0.45 \\
0.25\end{array}$ & Ir & $\mathrm{L}$ & & bluish \\
\hline 749.3 & 232246.2 & +122626 & $\begin{array}{l}0.60 \\
0.50\end{array}$ & $\begin{array}{l}0.40 \\
0.40\end{array}$ & $\mathrm{Ir}$ & $\mathrm{L}$ & F677-v4 & $V_{\mathrm{h}}=3664$ \\
\hline 605.2 & 232831.1 & +220957 & $\begin{array}{l}0.55 \\
0.45\end{array}$ & $\begin{array}{l}0.45 \\
0.40\end{array}$ & Ir & $\mathrm{L}$ & & arched, blue \\
\hline
\end{tabular}

Considering the distribution of nearby galaxies in approximately the same sky region, Nakanishi et al. (1997) determined the position of the Local Void to be about $\mathrm{RA}=21.0^{\mathrm{h}}, D=+12^{\circ}$, which is $32^{\circ}$ away from the above mentioned location. According to them the Local Void center corresponds to $V^{\mathrm{h}}=2500 \mathrm{~km} \mathrm{~s}^{-1}$. Apparently, we have here two different voids which are located near each other. To prevent confusions, we propose to call the nearest empty volume the "Hercules-Aquila Void", and the other one the "Pegasus-Delphinus Void".
Hoffman et al. (1992) have studied the Hercules-Aquila Void center in the HI line for low-mass gas clouds and HIrich dwarf galaxies. None were found within $2200 \mathrm{~km} \mathrm{~s}^{-1}$. For the mean Local Volume distance, $\sim 10 \mathrm{Mpc}$, a detection threshold in the $\mathrm{HI}$ line about $0.5 \mathrm{Jy} \mathrm{km} \mathrm{s}^{-1}$ corresponds to a hydrogen mass limit of $10^{7}$ solar masses.

\section{Concluding remarks}

Using the present data on redshifts for nearby galaxies we determined a more accurate center position and a 
boundary of the nearest void in Hercules-Aquila which has been found by Tully. Towards $\left\{\mathrm{RA}=18^{\mathrm{h}} 38^{\mathrm{m}}, D=+18^{\circ}\right\}$, the Local Void occupies an area $60^{\circ}$ in diameter and extends to $V_{0}=1500 \mathrm{~km} \mathrm{~s}^{-1}$. Our search for nearby dwarf galaxies made on the POSS-II films provided 78 objects, 22 of them being situated in the direction of the void. HI survey of these objects is in progress with the $100 \mathrm{~m}$ Effelsberg radio telescope.

The Hercules-Aquila Void is a perfect target for a search of very dwarfish (pygmy) galaxies, which may occurr in cosmic voids. This sky region looks to be very suitable for a systematic blind survey of dwarf irregular galaxies in $\mathrm{HI}$ down to $M_{\mathrm{HI}}=-13 \mathrm{mag}$, as well as lowmass gas clouds.

Acknowledgements. We have made extensive use of the Lyon-Meudon Extragalactic Database (LEDA, supplied by the LEDA team at the CRAL-Observatoire de Lyon), the NASA/IPAC Extragalactic Database (NED, which is operated by the Jet Propulsion Laboratory, Caltech, under contract with the National Aeronautics and Space Administration), and the Digitized Sky Survey (DSS-1) produced at the Space Telescope Science Institute under U.S. Goverment grant NAG W-2166. Our project is supported by INTAS-RFBR grant 95-IN-RU1390 and DFG grant KS 9112.

\section{References}

Balkowski C., Chamaraux P., 1983, A\&AS 51, 331 (BCh, 83) de Lapparent V., Geller M.J., Huchra J.P., 1986, ApJ 302, L1 Henning P.A., Kraan-Korteweg R.C., Rivers A.J., et al., 1998, AJ 115, 584

Hoffman G.L., Lu N.Y., Salpeter E.E., 1992, AJ 104, 2086

Huchtmeier W.K., 1998 (private information)

Impey C.D., Sprayberry D., Irwin M.J., Bothun G.P., 1996, ApJS 105, 209 (ISI, 96)

Joeveer M., Einasto J., Tago E., 1978, MNRAS 185, 357

Karachentsev I.D., 1994, Astron. Astrophys. Trans. 6, 1

Karachentseva V.E., Karachentsev I.D., 1998, A\&AS 127, 409 (Survey I)

Kirshner R.P., Oemler A., Schechter P.L., Shectman S.A., 1981, ApJ 248, L57

Nakanishi K., Takata T., Yamada T., Takeuchi T., et al., 1997, ApJS 112, 245

Paturel G., Fouque P., Bottinelli L., Gouguenheim L., 1992, Catalogue of Principal Galaxies, Lyon (PGC)

Roman A.T., Nakanishi K., Tomita A., Saito M., 1996, PASJ 48,679

Schombert J.M., Bothun G.D., Schneider S.E., McGaugh S.S., 1992, AJ 103, 1107

Schombert J.M., Pildis R.A., Eder J.A., 1997, ApJS 111, 233

Seeberger R., Huchtmeier W.K., Weinberger R., 1994, A\&A 286, 17

Tully R.B., 1988, Nearby Galaxy Catalog. Cambridge University Press 